\title{
TV/Series
}

$2 \mid 2012$

Les séries télévisées dans le monde : Échanges, déplacements et transpositions

\section{Take Me Back to Dear Old Blighty?}

Déplacements, hybridation et oscillation identitaires dans les séries humoristiques à contenu ethnique à la télévision outre-Manche

(1972-2011)

\section{Amandine Ducray}

\section{(2) OpenEdition}

12 Journals

Édition électronique

URL : http://journals.openedition.org/tvseries/1411

DOI : 10.4000/tvseries.1411

ISSN : 2266-0909

Éditeur

GRIC - Groupe de recherche Identités et Cultures

Référence électronique

Amandine Ducray, "Take Me Back to Dear Old Blighty? », TV/Series [En ligne], 2 | 2012, mis en ligne le 01 novembre 2012, consulté le 19 avril 2019. URL : http://journals.openedition.org/tvseries/1411

DOI : 10.4000/tvseries. 1411

\section{(c) (†) $\odot$}

TV/Series est mis à disposition selon les termes de la licence Creative Commons Attribution - Pas d'Utilisation Commerciale - Pas de Modification 4.0 International. 
Take Me Back to Dear Old Blighty?

Déplacements, hybridation et oscillation identitaires dans les séries humoristiques à contenu ethnique à la télévision outre-Manche (1972-2011)

Encore largement absentes du petit écran français, les séries humoristiques reposant sur des situations ethniques sont, en revanche, une constante en Grande-Bretagne depuis les années soixante-dix, lorsqu'apparaissent les premières sitcoms ethniques (black sitcoms), terme alors utilisé pour désigner des comédies de situation mettant en scène le contact entre des Britanniques issus de communautés minoritaires et des représentants de la " majorité » blanche. Dix à vingt ans plus tard, l'avènement d'un multiculturalisme télévisuel contribue à faire évoluer le paysage audiovisuel ; au face-à-face " Nous »/ « Eux » succèdent bientôt des séries comiques focalisées sur un univers minoritaire (all black sitcoms), britannique noir d'abord, puis britannique sud-asiatique. Au début du vingt et unième siècle, les réalisateurs, comédiens et scénaristes tendent cependant à abandonner progressivement le genre " comédie de situation » pour des formats sériels plus souples. On assiste alors à l'émergence d'un large panel de sketch shows qui donnent à voir, successivement, voire parallèlement, des identités " de trait d'union » fondamentalement hybrides, mais aussi plus exclusivement blanches. À l'heure où certains membres de la «majorité » dite «de souche" se sentent parfois "minoritaires" du fait du multiculturalisme, un vent de nostalgie parait en effet s'emparer d'humoristes et de spectateurs britanniques blancs en quête d'identité et qui semblent trouver dans la comédie un moyen détourné de renouer avec leurs racines. Adoptant une approche diachronique, le présent article s'attachera à observer les mutations des séries comiques à la télévision britannique dans leur approche de la question ethnique au sens large afin de voir comment, entre déplacements, hybridation et oscillation, elles ont pu refléter un questionnement durable, complexe et encore largement d'actualité en matière de relations raciales.

$\mathrm{E}$ n août 2010, le directeur du BBC College of Comedy, Micheal Jacob, jette un pavé dans la mare médiatique outre-Manche en déclarant que la BBC s'avère une impasse pour tout scénariste qui n'est ni blanc, ni issu de la classe moyenne ; la chaîne lance alors un concours pour faire émerger sa prochaine «sitcom multiculturelle ${ }^{1}$ ». Peu coûteuse, d'une structure simple et drôle quoiqu'empreinte de réalisme, la comédie de situation paraît en effet constituer un support privilégié pour aborder des préoccupations contemporaines parfois épineuses pour l'ensemble des téléspectateurs. Ainsi, dès 1972, sur fond d'immigration du Nouveau Commonwealth, Love Thy Neighbour (ITV, 1972-1976) promettait de "dédramatiser partiellement les

${ }^{1}$ Tamara Cohen, «BBC launches competition for multicultural writers as its sitcoms are branded 'too middle class'", Daily Mail online, 20 aout 2010 , http://www.dailymail.co.uk/tvshowbiz/article-1304608/BBC-competition-multiculturalwriters-sitcoms-branded-middle-class.html, consulté le 7 septembre 2011. 
relations raciales $^{2} »$ en suivant deux foyers voisins, blanc et noir 3 . Première d'une série de "sitcoms ethniques" (black sitcoms) en Grande-Bretagne, elle fut suivie de «sitcoms intégralement ethniques » (all black sitcoms), britanniques noires d'abord, dans les années quatre-vingt, puis britanniques sud-asiatiques, une tendance amorcée à la fin de la décennie. Témoignages d'une évolution des mentalités, ces étapes attestaient simultanément de la faculté du genre à perdurer grâce à sa " flexibilité idéologique 4 » .

Or, à l'aube du vingt et unième siècle, on observe une hybridation des formes et des contenus : la sitcom ethnique mute peu à peu en sketch show. Fondé sur l'enchaînement de saynètes comiques, ce format permet désormais de couvrir une palette d'individualités variées et intrinsèquement hybrides. Contemporaine d'un vaste débat sur l'identité nationale, une nouvelle génération de séries humoristiques voit pourtant bientôt le jour ; essentiellement focalisées sur des Blancs, dont elles interrogent en partie la spécificité ethnique, elles paraissent illustrer un nouveau déplacement, des " minorités » à la « majorité ».

Partant du postulat que la comédie fonctionne comme un miroir grossissant des transformations socioculturelles d'un pays donné, cet article s'attachera à analyser les mutations des séries télévisées humoristiques à contenu ethnique en Grande-Bretagne des années soixante-dix à nos jours, afin d'observer comment elles ont contribué à refléter un long et sinueux questionnement en matière de relations raciales.

\footnotetext{
${ }^{2}$ Phrase figurant sur la cassette VHS de Love Thy Neighbour, Saison 1 (ITV, 13 avril-25 mai 1972), distribuée par Pegasus Studio (2003).

3 À titre indicatif, la première sitcom ethnique française date seulement de 1990. Diffusée sur la toute récente M6 et co-écrite par Azouz Begag, La Famille Ramdam présentait le quotidien d'une famille française originaire d'Algérie. Comme Sixième Gauche (France 3, 1990), consacrée à deux familles voisines de palier - l'une «de souche ", l'autre d'appartenance maghrébine - elle ne connut qu'une seule saison. Malgré l'apparition récente de feuilletons à ancrage multiculturel, dont Plus Belle La Vie (France 3, 2004-), à ce jour, on ne relève aucune autre sitcom ethnique française, les chaînes de télévision préférant continuer d'importer des séries des États-Unis, une pratique lancée par TF1 dès 1982 avec Arnold et Willy (Diffrent Strokes, NBC, 1978-85 ; ABC, 1985-86). Pour plus de détails sur la diversité ethnique à la télévision française, voir par exemple les actes du colloque organisé le 26 avril 2004 par le Fonds d'action et de soutien pour l'intégration et la lutte contre les discriminations, Ecrans pâles? Diversité culturelle et culture commune dans l'audiovisuel, Paris, La Documentation française, 2004.

4 Jane Feuer note: « if we look at the sitcom in terms of what might be called its plot, we find little development or innovation. The situation has always been a simple and repeatable frame on which to hang all manner of gags, one-liners, warm moments, physical comedy and ideological conflicts. In fact, one could say that it has been the ideological flexibility of the sitcom that has accounted for its longevity. " Jane Feuer, "Situation comedy: part two », in The Television Genre Book, éd. Glen Creeber, Londres, British Film Institute, 2001, p. 69 [p. 69-70].
} 


\section{Les sitcoms ethniques des années soixante-dix : entre lieu commun et hétérotopie}

Née aux États-Unis dans les années trente, la comédie de situation (ou sitcom) se manifeste d'abord à la radio, puis à la télévision, et désigne :

une courte comédie narrative, sous forme de série, généralement d'une durée de vingt-quatre à trente minutes, et dont les personnages et leur environnement apparaissent de façon régulières.

Transmise chaque semaine, généralement en prime time, elle se distingue en outre du feuilleton par une structure narrative bouclée en fin d'épisode, ce qui en facilite la diffusion de façon aléatoire. Ses conventions génériques sont très codifiées, tant dans le déroulement de l'intrigue, dans le placement des caméras et le montage, qu'à travers l'utilisation de répliques destinées à être cultes (catchphrases) et de rires «en boîte" (canned laughter). Visant à susciter un sentiment communautaire chez le public, ceux-ci revêtent une importance capitale dans les sitcoms ethniques, où la frontière entre moquerie (laught at) et connivence (laugh with) est parfois ténue.

La première sitcom ethnique est elle-même américaine, il s'agit d'Amos 'n'Andy, dédiée à deux Afro-Américains quittant leur sud natal pour Chicago, puis pour New York. Diffusée à la radio à partir de 1928 et adaptée pour la télévision au début des années cinquante, la série prit néanmoins fin en 1953 suite à une campagne de la National Association for the Advancement of Coloured People 6 . Un an plus tard, elle connaît toutefois un nouveau souffle, en Grande-Bretagne, où elle est transmise sous sa forme originale jusqu'en 1957. Sa diffusion sur la $\mathrm{BBC}$ faisait écho à une réalité contemporaine : en 1948 avait été votée une loi qui accordait aux citoyens de l'ancien Empire le droit de s'installer, de travailler et de voter librement en territoire britannique (British Nationality Act).

C'est ensuite dans les années soixante, en pleine vague d'immigration du Nouveau Commonwealth, que démarre la production domestique de sitcoms couvrant, en partie, des considérations raciales. La première fut Till Death Us Do Part (BBC1, 1966-68 ; 1972 ; 197475), qui suivait le quotidien d'Alf Garnett, une figure d'ouvrier raciste campée par le comédien Warren Mitchell, dont l'ombre, comme on le verra, hante encore les comédies télévisées des années deux mille. L'intérêt ethnique y était cependant traité sous forme de trames secondaires. Car il faut attendre 1972 pour que la première sitcom

5 « The term 'sit-com' describes a short narrative-series comedy, generally between twentyfour and thirty minutes long, with regular characters and setting. " Steve Neale et Frank Krutnik, Popular Film and Television Comedy, Londres, Routledge, 1995 [1990], p. 233. ${ }^{6}$ Voir, par exemple, Darrell Y. Hamamoto, Nervous Laughter, Television Situation Comedy and Liberal Democratic Ideology, New York, Praeger, 1991 [1989], p. 42-43. 
ethnique britannique voie le jour. Diffusée sur ITV, Love Thy Neighbour se concentrait sur deux foyers mitoyens - blanc et noir - et plantait le décor à Twickenham, en banlieue de Londres, où les populations immigrées ont parfois effectivement choisi de s'installer. L'humour était à nouveau résolument ouvrier, en particulier à travers le héros blanc Eddie Booth (Jack Smethurst), un col bleu syndicaliste, lecteur assidu du tabloïd The Sun, amateur de pintes et de femmes plantureuses, mais aussi raciste latent. En transposant un univers familier du public et en le dotant de résonnances contemporaines, cette série semblait alors fonctionner comme une sorte d'hétérotopie, soit une forme d'utopie ancrée dans le réel ou, plutôt, dans un « effet de réel7 ». Voici comment Michel Foucault définit le concept d'« hétérotopie » :

des lieux réels, des lieux effectifs, des lieux qui sont dessinés dans l'institution même de la société, et [...] dans lesquels [...] tous les autres emplacements réels que l'on peut trouver à l'intérieur de la culture sont à la fois représentés, contestés et inversés, [...] bien que pourtant ils soient effectivement localisables [...] et je crois qu'entre les utopies et ces [...] hétérotopies, il y aurait sans doute une sorte d'expérience mixte, mitoyenne, qui serait le miroir. Dans le miroir, je me vois là où je ne suis pas, dans un espace irréel qui s'ouvre virtuellement derrière la surface, une sorte d'ombre qui me donne à moi-même ma propre visibilité $[\ldots]^{8}$.

Si elle s'applique à la plupart des fictions télévisées, qui existent à travers un lieu «localisable " où d'autres espaces peuvent être « représentés, contestés et inversés », ainsi qu'au rapport des téléspectateurs à un écran-miroir, à la fois substitut au quotidien et espace d'identification, cette citation semble aussi particulièrement convenir à Love Thy Neighbour, où le face-à-face ethnique s'appuie précisément sur la mitoyenneté des maisons, représentée dès l'ouverture du générique (voir figure 1) :

7 Le terme est celui de Roland Barthes, qui le définit de la sorte: «Sémiotiquement, le 'détail concret' est constitué par la collusion directe d'un référent et d'un signifiant; le signifié est expulsé du signe, et avec lui [...], la possibilité de développer une forme du signifié [...] C'est là ce que l'on pourrait appeler l'illusion référentielle. La vérité de cette illusion est celle-ci : supprimé de l'énonciation réaliste à titre de signifié de dénotation, le 'réel' y revient à titre de signifié de connotation ; car dans le moment même où ces détails sont réputés dénoter directement le réel, ils ne font rien d'autre, sans le dire, que le signifier [...]; autrement dit, la carence même du signifié au profit du seul référent devient le signifiant même du réalisme : il se produit un effet de réel [...] ». Roland Barthes, "L'Effet de réel ", in Littérature et réalité, éd. Gérard Genette et Tzvetan Todorov, Paris, Seuil, 1982, p. 88-89 [p. 81-90]. Emphases originales.

${ }^{8}$ Michel Foucault, «Des espaces autres » (conférence au Cercle d'études architecturales, 14 mars 1967), Architecture, Mouvement, Continuité, ${ }^{\circ} 5$, octobre 1984, p. 47 [p. 46-49]. Notre emphase. 


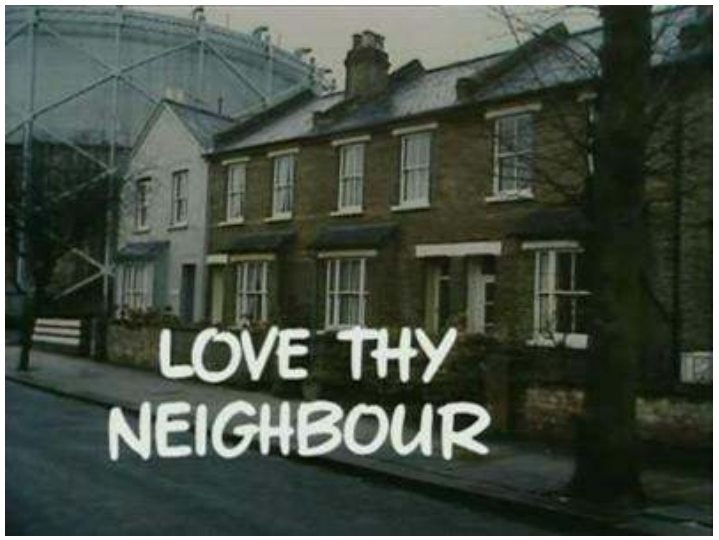

Fig. 1

Ce premier plan donne à voir une rue d'un quartier ouvrier britannique typique, reconnaissable à ses maisons de brique mitoyennes (terraced houses). L'usine à gaz à gauche de l'écran ainsi que l'arbre, dépourvu de feuilles, sur la droite, contribuent à traduire un quotidien ordinaire et prosaïque. D'un point de vue esthétique, on pourrait être tenté d'y voir une tentative télévisuelle de "réalisme social ». En termes scénographiques, la mitoyenneté des maisons indiquait, en tout cas, qu'une importance significative était accordée au pas de porte, sur lequel se clôt, semaine après semaine, le générique d'ouverture.

Point de jonction entre espace public et espace privé et entre des univers domestiques distincts, dès le premier épisode, cet entredeux servait à annoncer l'arrivée de voisins originaires de Trinidad : c'est en allant chercher son lait devant chez elle que Joan Booth (Kate Williams), remarquait le camion des déménageurs9. Plus tard, c'est en découvrant sa voisine noire, Barbie Reynolds (Nina Baden-Semper), penchée de l'autre côté de la haie, révélant malgré elle la partie la plus charnue de son anatomie, qu'Eddie Booth, debout sur le seuil de son domicile, renonçait à déménager, une décision qui consacrait la restauration de la situation de base du programme - la cohabitation entre Britanniques "de souche» et représentants d'un groupe ethnique minoritaire ${ }^{10}$. Perçue comme de la promiscuité par le Blanc, la proximité des foyers était un ressort narratif et comique essentiel dans Love Thy Neighbour. Elle suscitait pléthore de fantasmes, notamment celui d'une hypersexualité noire, d'où l'emploi d'une

${ }_{9}^{9}$ Saison 1, épisode 1 (ITV, 13 avril 1972).

${ }^{10}$ Deux épisodes plus tard, Eddie s'efforce à nouveau, en vain, de mettre un terme à cette situation en faisant circuler une pétition visant à chasser le couple noir de son quartier. Saison 1, épisode 3 (27 avril 1972). 
réplique récurrente chez Eddie, «Belts up! », qu'il hurle, toujours à tort, à travers la cloison mitoyenne ${ }^{11}$, le domicile jouxtant le sien devenant en quelque sorte cet « espace irréel qui s'ouvre virtuellement derrière la surface » tel que l'évoque Michel Foucault.

La mise en scène d'un face-à-face se poursuivait dans le décor des foyers; là où les Booth affichaient des tableaux de beefeaters et d'un clipper ${ }^{12}$, les Reynolds préféraient ceux de plages et de femmes noires dénudées, sorte de contrepoint antillais à une imagerie nationale éculée. Le pas de porte apparaissait par conséquent comme le seul lieu commun possible entre ces deux communautés, car même l'usine sidérurgique où travaillaient les deux hommes ne servait qu'à accentuer une " comédie de frustration " centrale à d'autres sitcoms non spécifiquement ethniques ${ }^{13}$. Combiné au principe du huis clos, également caractéristique du genre, ceci revêtait toutefois une valeur singulière dans Love Thy Neighbour ; malgré un rapprochement entre protagonistes féminins, le conflit systématique entre Eddie Booth et son voisin et collègue noir Bill Reynolds (Rudolph Walker) revenait à présenter l'harmonie interraciale comme vouée à l'échec. Les oppositions binaires y abondaient: Noir/Blanc, Conservateur/ Travailliste, sportif/pantouflard, etc. Car le duo comique fonctionnait selon le principe d'un miroir inversé ; la représentation de l'« Autre » n'étant guère plus qu'une représentation du « Soi » en négatif, ou, pour reprendre l'observation foucaldienne, « une sorte d'ombre qui [lui] donne à [lui]-même [sa] propre visibilité », mais aussi, par procuration, celle du public blanc.

Le sentiment d'une frontière inamovible entre un "Nous » britannique blanc et un «Eux » considéré comme exogène était, en effet, d'autant plus aigu dans la série que Bill, certes doté d'un droit de réponse face à la litanie de stéréotypes vociférée par Eddie, était souvent éclipsé par la truculence de ce dernier, voire par ce que d'aucuns pouvaient à l'époque considérer comme son franc-parler. Le héros ne s'autorisait-il pas à dire tout haut ce que certains spectateurs pensaient tout bas? De fait, si Love Thy Neighbour renvoyait à une

${ }^{11}$ Il s'agit d'un jeu de mot sur l'impératif « belt up! » («bouclez-la! ») auquel Eddie ajoute la marque du pluriel comme pour signifier aux voisins, littéralement, de remonter leur pantalon/ceinture (belts).

${ }_{12}$ Vraisemblablement une déformation de l'ancien français «buffetier », beefeater est le nom donné aux gardes de la Tour de Londres. L'image de clippers, de la même façon, illustre une page de l'histoire britannique, celle de l'Empire, où ces bateaux à voile, dont le célèbre Cutty Sark, servaient de trait d'union entre la Grande-Bretagne, à l'apogée de sa puissance commerciale, et ses colonies.

13 « a comedy of frustration », James Baker, Teaching TV Sitcom, Londres, British Film Institute, 2003, p. 40. Mick Eaton note par ailleurs : « The necessity for the continuity of character and situation from week to week allows for the possibility of comedy being generated by the fact that the characters are somehow stuck with each other [...] It is as if the formal necessities of the series provide the existential circle from which the characters cannot escape. » Mick Eaton, «Television Situation Comedy », Screen, vol. 19, n ${ }^{\circ}, 1978$ 79, p. 74-75 [p. 61-89]. 
situation susceptible d'être vécue par quelques-uns des spectateurs, l'opinion du Blanc vis-à-vis de son voisin noir pouvait faire écho à un credo jusqu'alors cantonné aux partis d'extrême droite et auquel le Conservateur Enoch Powell donna une nouvelle impulsion quelques années avant les débuts de la série ${ }^{14}$. En avril 1968, cette figure politique controversée prononça un discours sur les dangers de l'immigration, bientôt connu en tant que «Discours des rivières de sang ». D'un impact considérable sur l'ensemble des citoyens britanniques ${ }^{15}$, cette allocution marqua un point de non retour dans l'histoire des relations raciales; elle eut pour effet, chez certains Blancs, de conforter, voire de générer, un sentiment de peur, et contraignit de facto les deux principaux partis politiques, Conservateurs et Travaillistes, à une fermeté accrue en matière d'immigration ${ }^{16}$.

Or, en dépit d'une volonté de "dédramatiser partiellement les relations raciales ${ }^{17}$ ", Love Thy Neighbour recyclait plusieurs préjugés. Bien qu'assumées par un héros aussi manifestement borné et grotesque qu'Eddie Booth, des affirmations comme « Ce sont tous des violeurs », " ils baisent comme des lapins », " y'en a un qui débarque et toute la smala suit », ou encore, dans un amalgame flagrant, «ils puent le curry », pouvaient alors peut-être, chez certains spectateurs, entrer en résonnance avec des idées reçues quoiqu'infondées autrement dit des lieux communs - sur les Noirs, la sitcom jouant manifestement sur l'ambivalence du rire vis-à-vis du Blanc. Ce fut, en

\footnotetext{
14 Lors d'un entretien avec Vince Powell, co-scénariste de Love Thy Neighbour, quelques semaines seulement après les attentats londoniens de 2005, ce dernier a d'ailleurs incidemment remarqué à propos d'Enoch Powell : «He has been proved right now, unfortunately ». Amandine Ducray, Entretien avec Vince Powell, Londres, $1^{\mathrm{er}}$ août 2005, in Amandine Ducray, «Les Sitcoms ethniques à la télévision britannique (1972-1998)» (Appendices, vol. 2), Thèse de doctorat dirigée par Renée Dickason et soutenue à l'Université de Caen le 5 décembre 2005, p. 594 [p. 589-598].

${ }^{15}$ Certes énoncé devant une centaine de personnes seulement, lors de l'assemblée générale du Parti Conservateur des West Midlands, et en l'absence de caméras de télévision, ce discours, d'après un sondage Gallup, fut bientôt connu de $86 \%$ de la population britannique adulte qui, en l'espace de deux jours, en avait entendu des bribes. Voir Sarita Malik, Representing Black Britain: Black and Asian Images on Television, Londres, Sage, 2002, p. 45

${ }_{16}$ Ce climat politique complexe a été résumé dans un aphorisme forgé par un député travailliste: "Without integration limitation is inexcusable, without limitation integration is impossible. » Roy Hattersley cité dans Andrew Geddes, The Politics of Immigration and Race, Manchester, Baseline Books, 1996, p. 49. La deuxième moitié des années soixante et les années soixante-dix furent de fait marquées par un jeu de bascule entre mesures favorables à l'intégration des minorités ethniques déjà présentes sur le sol britannique (les Race Relations Acts de 1965, 1968 et 1976) et contrôles d'immigration de plus en plus draconiens à l'égard de leurs compatriotes (Commonwealth Immigrants Acts de 1962 et 1968 et Immigration Act de 1971). Pour plus de détails, voir par exemple Bob Hepple, "Have Twenty-five Years of Race Relations Acts in Britain been a Failure? ", in Discrimination: The Limits of the Law, éd. Bob Hepple et Erika Szyszczak, Londres, Mansell, 1992, p. 284-291.
}

${ }_{17}$ Voir note 2 du présent article. 
tout cas, le point de vue de la Race Relations Board, organisme chargé de promouvoir l'égalité raciale en Grande-Bretagne ${ }^{18}$, qui reprocha notamment aux scénaristes la répétition des termes coon, sambo ou golliwog - soit autant de variations terminologiques du français « bamboula ». En 1976, la société de production Thames Television, avec laquelle collaborait la chaîne ITV, décida, par conséquent, de ne pas renouveler Love Thy Neighbour ${ }^{19}$. Un an après celui de Till Death Us Do Part, son arrêt semble ainsi avoir signé la fin d'une époque où l'on pouvait rire, en toute ambigüité, du racisme affiché par des héros blancs.

\section{Le tournant multiculturel des années quatre-vingt et quatre-vingt-dix : vers une identité « de trait d'union »?}

La seconde moitié des années soixante-dix et les années quatrevingt voient un changement profond dans l'appréhension des relations raciales outre-Manche, y compris sur le petit écran. Publié en 1977, le rapport Annan exige du service public de télévision de s'adresser à tous, quel(le) que soit leur phénotype, leur culture ou leur religion ${ }^{20}$. Signe d'une sensibilité pluraliste émergente, en novembre 1982, il déboucha sur la création d'une quatrième chaîne destinée, entre autres, à cibler les spectateurs minoritaires. La question d'un portrait global de la société britannique apparaissait alors d'autant plus d'actualité qu'en avril 1981, quelques mois avant le lancement de Channel Four, avaient éclaté des émeutes raciales, dans le quartier londonien de Brixton d'abord, puis à travers plusieurs autres métropoles. Publié consécutivement à ces « Troubles », le rapport Scarman comportait la première mention officielle d'un « racisme institutionnel » britannique, qu'il appliquait à la police, et engageait les médias à chercher davantage d'équilibre dans la couverture de ce type d'événements ${ }^{21}$. Quatre ans plus tard, le rapport Swann sur l'éducation aboutissait à un constat similaire, relevant à nouveau un devoir

\footnotetext{
${ }^{18}$ La Race Relations Board a été créée en 1965, lors du vote du premier Race Relations Act. Notons sa mention dans le premier épisode de Love Thy Neighbour. À l'issue de sa rencontre avec Bill, Eddie décide de téléphoner à cet organisme. Ignorant tout de ses véritables fonctions, il se montre grossier et insultant : «I wish to make a complaint [...] Pardon? Am I coloured? Yes, white all over and I'm proud of it! I wish to make a complaint against a nig nog [...] Oh, very well. A coloured gentleman then [...] What has he done? He's moved next door, that's what he's done! » Saison 1, épisode 1 (13 avril 1972).

19 Pour plus de détails, voir Amandine Ducray, Entretien avec Vince Powell, op. cit., p. 591 et passim. En complément, voir Michael Anwar et Anthony Shang, Television in a MultiRacial Society, A Research Report, Londres, Commission for Racial Equality, 1982, p. 2021.

${ }^{20}$ Lord Annan, Report of the Committee on the Future of Broadcasting under the Chairmanship of Lord Annan, Cmnd 6753, Londres, HMSO, 1977.

${ }^{21}$ Lord Scarman, The Brixton Disorders 10-12 April 1981, Cmnd 8427, Londres, HMSO, 1981.
} 
médiatique par rapport aux minorités ${ }^{22}$. Les chaînes publiques ne tardèrent pas à réagir et, parallèlement à Channel Four, la BBC et ITV commencèrent à réfléchir aux moyens de refléter la diversité23.

La diffusion de sitcoms intégralement ethniques peut être lue comme une étape de cette prise de conscience. Comme dans les années cinquante, un premier réflexe fut de se tourner vers les États-Unis et c'est, en fait, dès 1976, l'année où cesse Love Thy Neighbour, qu'ITV programme The Fosters (1976-77), inspirée de Good Times (CBS, 197479) et qui transposait une famille afro-américaine en une famille britannique d'origine guyanaise ${ }^{24}$. Cette sitcom ne dura qu'une saison, ce qui s'explique vraisemblablement par sa précocité et, malgré un effort d'adaptation, par son manque de spécificité culturelle britannique, les scripts étant largement calqués sur les originaux. D'après Norman Beaton, qui y incarnait le père de famille,

The Fosters a été un transfert. Elle n'avait strictement rien à voir avec la situation endémique des Antillais de Grande-Bretagne ${ }^{25}$.

Le comédien noir compare The Fosters à Desmond's (C4, 1989-94), dans laquelle il a également joué, et relève une " progression » d'une sitcom à l'autre :

Écrite par un scénariste antillais, Trix Worrell, Desmond's se concentre sur de vraies personnes ${ }^{26}$.

\footnotetext{
${ }^{22}$ Lord Swann, Education for All: Report of the Committee of Inquiry into the Education of Children from Ethnic Minority Groups, Cmnd 9453, Londres, HMSO, 1985.

${ }^{23}$ Voici ce que déclarait, par exemple, un membre de la BBC en 1986, lors d'une conférence sur les implications du rapport Swann : «We in broadcasting have yet fully to work out, and to implement [...] what is responsible programming in and for a multicultural and multiracial society still characterised - as the Swann Report confirms - by continuing prejudice and discrimination against black people. [...] As with most schools and colleges, the BBC is not yet equipped with those black staff in senior roles needed both to fulfil policies of equal opportunity and to bring a black perspective to its decision-making. While these difficulties are being worked upon, teachers [...] need to give priority to ways of drawing the attention of pupils to the nature of the media influences upon them [...]. * Rapport de la conférence "Swann and the Global Dimension », Université de Bristol, janvier 1986, cite dans The Black and White Media (Show) Book, Handbook for the Study of Racism and Television, éd. John Twitchin, Stoke-on-Trent, Trentham Books, 1992 [1988], p. 195-196. Notons, cependant, le commentaire du Directeur général de la BBC Greg Dyke (2000-4), peu de temps après sa nomination : «The BBC is an horrendously white organisation ». Au seuil du nouveau millénaire, les recommandations du rapport Swann n'avaient manifestement toujours pas été mises en place de façon concrète. Voir «Greg Dyke makes ethnic diversity a top priority at the BBC », BBC News Release, 7 avril 2000.

${ }^{24} \mathrm{La}$ première all black sitcom de la BBC date, quant à elle, seulement de 2003. Il s'agit de The Crouches (BBC1, 2003-5), dont la première saison provoqua un tollé en raison du choix d'un scénariste blanc, l'Écossais Ian Pattison, remplacé par Liselle Kayla, d'origine jamaïcaine, dans la seconde saison.

25 "The Fosters was a transfer that had really nothing whatsoever to do with the endemic situation of West Indians in this country. " Norman Beaton in Black and White in Colour: Black People in British Television Since 1936, éd. Jim Pines, Londres, British Film Institute, 1992, p. 118

26 "Desmond's is a progression from The Fosters in the sense that it is written by a West Indian writer, Trix Worrell, and is about real people. » Ibid.
} 
De même que l'ancrage de Love Thy Neighbour traduisait une forme de britannicité topographique, Desmond's donnait à voir, dès le générique, un paysage emblématique du ferment multiculturel de la fin des années quatre-vingt. La série avait pour décor Peckham, un quartier du sud-est de Londres à forte densité de populations noires, et combinait deux sphères, domestique et professionnelle, une fusion transcrite par son titre, un cas possessif désignant la boutique tenue par les protagonistes principaux. Après plusieurs plans urbains, plus ou moins rapprochés, sur des passants, majoritairement noirs, le générique d'ouverture s'achevait d'ailleurs sur un plan de l'échoppe, devant laquelle se tenaient le héros et sa femme (voir figure 2).

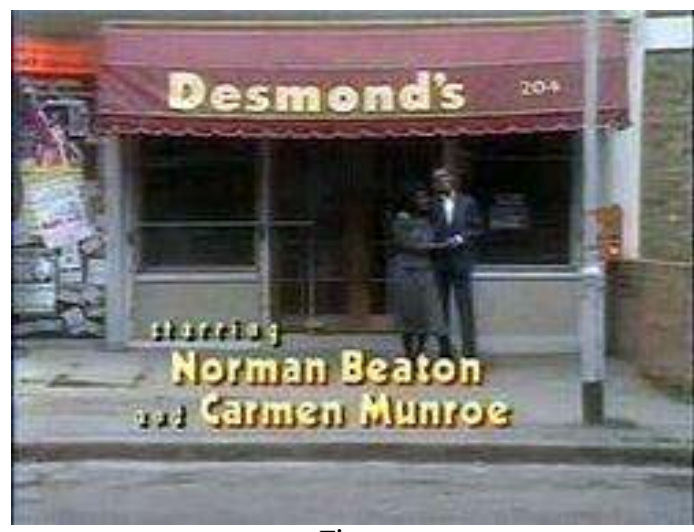

Fig. 2

Centrée sur une famille noire (les Ambrose), elle se focalisait en effet simultanément sur les activités d'un coiffeur pour hommes (Desmond Ambrose) dont les clients étaient eux-mêmes tous noirs. Au niveau narratif, le repli communautaire qui aurait pu découler de cette situation était nuancé par la façon dont chaque génération négociait avec le modèle culturel dominant. Tandis que Sean (Justin Pickett), le benjamin, puisait son inspiration dans la diaspora afro-américaine, à travers le rap et le break-dancing, et que son frère Michael (Geff Francis), banquier de profession, était jugé trop « britannique » par ses proches, leur père n'avait de cesse d'évoquer son « retour au pays », une photographie de son «lopin de terre » en Guyane à la main²7. À

${ }^{27}$ Ceci renvoyait à une réalité sociale : des années soixante-dix aux années quatre-vingt, près d'un Britannique antillais sur cinq a effectivement choisi de rentrer « au pays » à l'âge de la retraite. Voir Robin Cohen, Frontiers of Identity, The British and the Others, Londres, Longman, 1994, p. 66-67. 
une binarité «Nous»/«Eux» avait donc ici été substituée la focalisation sur un "Nous» complexe, oscillant entre un «ici et maintenant » et un « ailleurs jadis ${ }^{28}$.

Bien que dédiée à un univers noir, la série comptait, en outre, deux Blancs ; la «majorité » blanche, devenue minoritaire, faisait parfois l'objet de sarcasme. Lorsque le salon de Desmond risque d'être racheté, Louise (Lisa Geoghan), une amie blanche de la famille, se lance par exemple dans un dithyrambe du lieu, qu'elle érige en "bastion de défense des droits sociaux», concluant qu'il faut " défendre les petits », remarque peu appréciée du coiffeur à en juger par le plan de réaction qui suit ce monologue ${ }^{29}$. Car, quoique favorable aux minorités, son discours plaquait sur la communauté de Desmond's une lecture politique toute personnelle. En dépit d'une bonne volonté indéniable, Louise était, en effet, comme figée dans une posture d' «anti-raciste de gauche » somme toute peu nuancée ${ }^{\circ}$. Tony (Dominic Keating), l'assistant blanc de Desmond, était quant à lui féru de boxe et de jazz et, doté d'une gestuelle et d'un argot "de Noir », s'efforçait constamment d'être intégré, pour ne pas dire assimilé, au milieu dépeint dans la série. La présence de ces personnages dans cette sitcom majoritairement ethnique avait plusieurs fonctions : inverser une relation jusqu'ici conventionnelle à la télévision britannique Louise et Tony devenant ainsi quasiment des "Blancs de service " (token Whites) ${ }^{31}$; témoigner d'un rapprochement interracial; et faciliter l'exploration de sujets sensibles pour les Britanniques noirs sans aliéner l'ensemble du public. Desmond's s'autorisait, en effet, quelques commentaires d'ordre politique, en particulier par le truchement de Porkpie (Ram John Holder), le meilleur ami du coiffeur $^{32}$. Dans l'épisode consacré à un rachat éventuel du salon, il

\footnotetext{
${ }^{28}$ Notons aussi, à ce propos, l'existence d'une rivalité taquine entre Desmond et Matthew, un ami africain. Lorsqu'il joue aux dominos avec lui, Desmond, agacé par sa lenteur, commente par exemple : "Don't you play dominoes in Africa? ». Matthew réplique alors : "In Gambia we play Trivial Pursuit », suggérant une supériorité intellectuelle des Africains sur les Antillais. Saison 1, épisode 1, « The French Lesson » (C4, 5 janvier 1989).

29 « a place of social vindication »; «We'll support the little man ». Saison 1, épisode 2, «Short and Curlies » (12 janvier 1989).

${ }^{30}$ Pour une critique de ce type de discours, voir par exemple Paul Gilroy, « Two sides of anti-racism ", in There Ain't No Black in the Union Jack, Londres, Routledge, 2002 [1987], p. 146-200. On y relève par exemple, dans une phrase qui paraît transposable au personnage de Louise dans Desmond's: "the attempt to make 'race' always already a meaningful factor, in other words to racialize social and political phenomena, may be itself identified as part of the 'race' problem ». Ibid., p. 150.

${ }^{31}$ Durablement appliqué aux minorités ethniques dans les fictions télévisées, le tokenism consiste à intégrer quelques personnages secondaires, voire de simples figurants, à la trame de programmes majoritairement blancs. En 1983, Equity, le principal syndicat d'acteurs britanniques, s'opposa officiellement à cette pratique. Equity's Policy Statement on Integrated Casting, Londres, Equity, 1983

${ }^{2}$ Suite au décès soudain de Norman Beaton (Desmond Ambrose), Channel Four lance d'ailleurs Porkpie (1995-96), une série dérivée (spin-off) où l'ami de Desmond changeait subitement de train de vie en gagnant au loto.
} 
rétorquait à Desmond, un temps enorgueilli par son statut de travailleur indépendant :

Hormis des épiceries de quartier, des stands de vente à emporter, des restos, des fish and chips ou des kebabs, hormis un salon de coiffure à Peckham, c'est quoi notre activité économique?

Et le coiffeur de répondre :

Eh bien, d'après ce qu'en disent les gens, il semblerait qu'on ait un monopole dans les agressions et les délits sur la voie publique 33 .

L'observation tendait à indiquer que la perception des minorités avait peu évolué depuis la publication des rapports Scarman et Swann. Montrer un personnage tel que Porkpie, taciturne, en situation de précarité et doté d'un penchant pour la bouteille, favorisait par ailleurs l'expression d'une réalité vécue par d'autres immigrés de "première génération ", amers par rapport à leur expérience britannique 34 .

Du fils aîné Michael, dont la réussite sociale faisait débat, à Porkpie, parangon de pauvreté subie, représenter une communauté hétérogène semble avoir évité à Desmond's les écueils rencontrés par No Problem! (C4, 1983-85), une all black sitcom articulée autour d'une cellule familiale réduite aux enfants, leurs parents étant rentrés en Jamaïque. D’après Paul Gilroy, ceci « revenait à présenter le modèle familial noir comme a priori incomplet et défaillant 35 », le chercheur et activiste noir écrivant même que la série reposait sur une notion alors controversée: le rapatriement volontaire ${ }^{36}$. Inventée par les Britanniques sud-asiatiques Farrukh Dhondy et Mustapha Matura, No

\footnotetext{
33 PORKPIE. Cornershops, takeaways, restaurants, fish 'n' chips, doner kebabs. Apart from a barber-shop in Peckham, what business do we own?

DESMOND. Well, judging from the people, we seem to have a monopoly on muggings and street-crimes. (Saison 1, épisode 2)

34 "Porkpie's Birthday » est sans doute l'épisode le plus représentatif à cet égard. Lors d'une visite de Gloria, la fille des Ambrose, le public y découvrait le domicile de Porkpie, un studio aux allures de garçonnière dans une tour HLM. Ce dernier commentait : " It's funny, I worked hard all my life and what me have to show for it [sic]? A flat on the $7^{\text {th }}$ floor in the worst estate in Peckham! », Saison 1, épisode 5, "Porkpie’s Birthday » (2 février 1989). Sur les minorités ethniques et la pauvreté dans la Grande-Bretagne contemporaine, voir par exemple Kaushika Amin et Carey Oppenheim, Poverty in Black and White: Deprivation and Ethnic Minorities, Londres, Child Poverty Action Group/Runnymede Trust, 1992. 35 « The absence of the parents means that No Problem! presents the black family a priori incomplete and deficient. » Paul Gilroy, " $\mathrm{C} 4$ Bridgehead or Bantustan? », Screen, juilletoctobre 1983, reproduit in Black Images in British Television, The Colour Black, éd. Therese Daniels et Jane Gerson, Londres, British Film Institute, 1989, p. 41 [p. 40-43]. ${ }^{36}$ Jusqu'alors défendue par une poignée d'hommes politiques, l'idée d'un rapatriement volontaire en partie financé par l'État prit un tournant historique en 1983, l'année où démarra No Problem!, quand des députés Conservateurs, lors de la conférence annuelle de leur parti, firent pression sur le gouvernement Thatcher pour abonder dans ce sens. Le Ministre de l'Intérieur, David Waddington, s'y opposa farouchement, déclarant: "The government is not in the business of telling people who have made their lives here, who perhaps even have become British citizens: 'You are unwelcome. Here is some money. Clear off!' », David Waddington, Financial Times, 14 octobre 1983, cité dans Cohen, p. 66.
} 
Problem! ne collait en outre vraisemblablement pas au plus près d'une réalité noire. En 1984, devenu directeur des programmes multiculturels sur Channel Four (1984-97), Farrukh Dhondy décida précisément de créer la première sitcom britannique sud-asiatique, Tandoori Nights (C4, 1985-87), consacrée à la rivalité entre deux restaurants indiens. Son succès fut pourtant également mitigé et il fallut attendre plus d'une décennie pour que l'humour britanniquesud-asiatique s'épanouisse à la télévision.

Diffusé sur BBC2 de 1998 à 2000, un sketch show intitulé Goodness Gracious $\mathrm{Me}$ vit le jour à une époque où la production artistique de Britanniques d'origine indienne ou pakistanaise était en plein essor et parfois désignée sous l'étiquette Bollywood-in-Blighty ${ }^{37}$. Qualifiée de «lubrifiant des relations raciales britanniques» par le Premier ministre Tony Blair ${ }^{8}$, cette série semblait transcender la question des identités britanniques et/ou sud-asiatiques pour afficher une identité de trait d'union (hyphenated), britannique-sud-asiatique, voire quasiment britannique, son titre renvoyant au leitmotiv du gourou interprété par Peter Sellers, grimé en Indien, dans le film The Millionairess (réal. Anthony Asquith, 1960)39. Dès l'un des premiers sketches, où un groupe d'Indiens se rendait dans un restaurant anglais, la sitcom avait certes recours à une comédie de " racisme inversé »40.

De même, le sketch " High and Blighty », un pseudo "docusoap sur une famille anglaise type prise sur le vif 41 » à destination d'un

37 Issu de la contraction de « Bombay » et de « Hollywood », le terme « Bollywood » renvoie à l'industrie cinématographique indienne, tandis que le terme Blighty peut être défini ainsi : " an informal and often affectionate term for Britain or England, chiefly as used by soldiers of the First and Second World Wars. The term was first used by soldiers serving in India, and is an Anglo-Indian alteration of Urdu bilāyatī 'foreign, European', from Arabic wilāyat, wilāya 'dominion, district'. » Elizabeth Knowles (éd.), The Oxford Dictionary of Phrase and Fable, Oxford, New York, Oxford University Press, 2001, p. 120.

38 " the oil that lubricates the wheels of race relations in this country. » Tony Blair, cite de mémoire par Sharat Sardana, co-scénariste de Goodness Gracious Me. Voir Amandine Ducray, «Ethnic Minorities, Comedy and British Television. Interview with Sharat Sardana. London, Friday 26 of November 2004", Revue LISA/LISA e-journal, http://lisa.revues.org/index664.html, consulté le 2 août 2011.

39 Interrogé sur la teneur de l'humour dans Goodness Gracious Me, Sharat Sardana répondit d'ailleurs spontanément: "British. Very old-fashioned British comedy. Purposely. " Il ajouta cependant : "The thing that makes it different is that it is an Asian sketch show... And that is what we wanted to do. It did not occur to me, or to my writing partner Richard Pinto, who is Portuguese, to do something just Asian. It would have to be just funny, it did not matter what culture it came from... », Ibid. Notons par ailleurs que le pilote de la série s'intitulait « Peter Sellers is Dead », qui renvoyait à l'arrêt du procédé du blacking up (le fait de se grimer en Noir ou en Indien), procédé employé à plusieurs reprises par le comédien anglais au cours de sa carrière.

${ }^{40}$ Saison 1, épisode 1 (BBC2, 12 janvier 1998). Le producteur Anil Gupta note : "One of our first sketches was 'Going for an English' (a group of Asians behave badly in an English restaurant - in the style of boozy lads at a curry house). We suggested that people tend to be a bit rude in Indian restaurants without saying it. " The Journal of the Royal Television Society, «A new kind of laughter », janvier 2000, p. 24.

41 " A fly-on-the-wall docu-soap about a typical English family », Saison 2, épisode 6 (17 décembre 1998). Commentaire en voix off. 
public indien, retournait le miroir, pour ainsi dire, en direction des Blancs. Outre un patriotisme ostensible (voir figure 4), les comédiens Meera Syal, Nina Wadia, Kulvinder Ghir et Sanjeev Bhaskar faisaient montre d'une vulgarité sans borne, s'invectivant et s'insultant à tout bout de champ. De plus, tous y étaient talqués « en Blanc », Bhaskar jouant un père qui, derrière ses lunettes et son costume, ressemblait en tous points à Alf Garnett, le héros raciste de Till Death Us Do Part, diffusée sur la BBC plus de trente ans auparavant (voir figure 3).

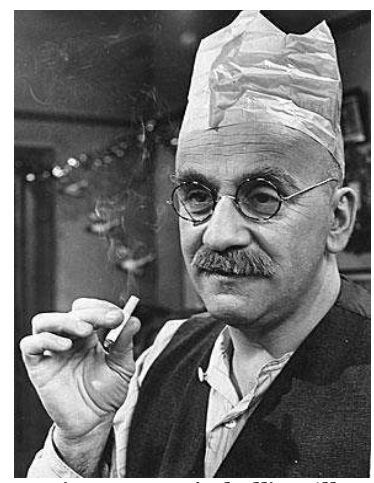

Fig. 3 : Alf Garnett (Warren Mitchell), Till Death Us Do Part Saison 2, épisode 1 (BBC1, 26 décembre 196642)

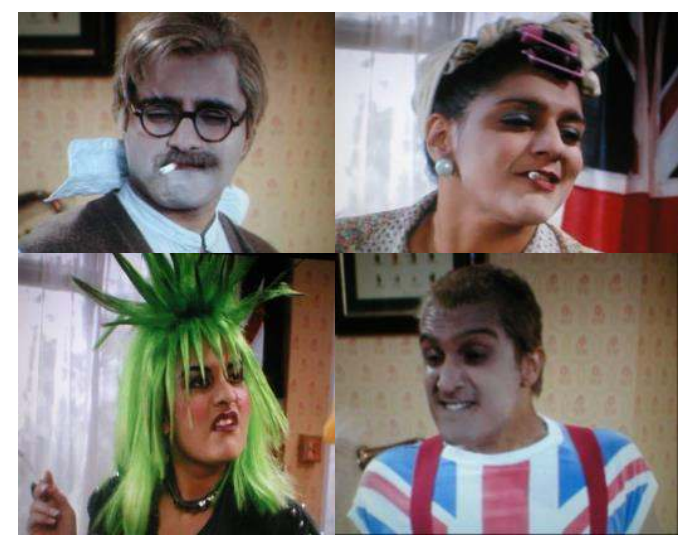

Fig. 4 : Goodness Gracious Me, sketch « High and Blighty », Saison 2, épisode 6 (17 décembre 1998)

\footnotetext{
${ }^{42}$ Le chapeau que porte le comédien Warren Mitchell est à relier à la date de diffusion de l'épisode, le lendemain de Noël. Porter ce type de couronne en papier relève en effet d'une tradition britannique en cette période de l'année.
} 
Au-delà de la parodie du père en avatar du héros de Till Death Us Do Part, de la mère en mé(na)gère arborant fichu, bigoudis, cigarette et maquillage appuyé, et des deux enfants en punks, l'intericonicité, en particulier par le truchement d'Alf Garnett, relevait vraisemblablement d'un clin d'œil de la jeune génération britannique sud-asiatique à l'ancienne génération de scénaristes et de comédiens blancs 43 .

Vu son format, le sketch show privilégiait une nomenclature quelque peu normative. Il y existait des Indiens " excessivement indiens 44 » (Mr 'Everything-Comes-From-India', Uncle 'You-ShouldHave-Come-To-Me' ou Mrs 'I-Can-Make-It-At-Home-For-Nothing'45) ; des Indiens « excessivement britanniques » (Les Kapoor " prononcez Cooper »), ainsi que divers Blancs entichés d'un exotisme indien fantasmé46. On comptait toutefois des héros hybrides tels les Bhangra Muffins, dont le nom évoque d'emblée une identité de trait d'union ${ }^{47}$. C'est bien en enchaînant une pluralité de héros, de thèmes, de lieux et d'époques que Goodness Gracious $M e$ embrassait une identité britannique enrichie d'emprunts culturels indiens; le bhangra y côtoyait les morris dances, les saris les Doc Martens et, comme chez la réalisatrice Gurinder Chadha, Pride and Prejudice (Jane Austen, 1813) était transposé en mode bollywoodien ${ }^{48}$.

43 Lors d'un entretien, Sharat Sardana fit d'ailleurs montre d'une certaine fierté en racontant que lors d'une soirée organisée par la BBC, il avait été félicité par Jimmy Perry et David Croft, les scénaristes de It Ain't Half Hot, Mum (BBC1, 1974-81), une sitcom ethnique des années soixante-dix qui avait pour toile de fond le Raj britannique. Voir Amandine Ducray, «Ethnic Minorities, Comedy and British Television. Interview with Sharat Sardana. London, Friday 26 of November 2004 ", op. cit.

44 Cette typologie est inspirée de notre entretien avec Sharat Sardana. Ibid.

45 Bien que non employés par les personnages eux-mêmes, ces surnoms, tirés de leurs leitmotivs (catchphrases), se sont vite imposés auprès du public britannique.

${ }^{46}$ On pense notamment aux clients crédules d'un gourou arnaqueur récurrent dans la série, qui, au cours de ses diverses séances, prétend leur raconter l'H/histoire des divinités hindoues et récite en fait, devant un auditoire imperturbable, des paroles de chansons à succès, par exemple le titre du groupe Queen, « Bohemian Rhapsody ». Saison 2, épisode 3 (27 novembre 1998).

47 Le bhangra désigne une musique du Punjab tandis que bhangramuffin est calquée sur l'expression raggamuffin, qui couvre un mouvement musical noir. On pourrait aussi y voir un clin d'œil au gâteau du même nom, très répandu dans les pays anglo-saxons, ce qui témoignerait d'une nouvelle fusion culturelle. Ainsi que le relève Marie Gillespie, le duo fut particulièrement populaire auprès des adolescents britanniques, toutes origines confondues, qui reprenaient leur formule Kiss my Chuddies!. Voir Marie Gillespie, « From Comic Asians to Asian Comics: Combating Intolerance through TV Comedy ", Communication présentée à Stockholm, Forum international sur l'intolérance (2930 janvier 2001), http://2001.stockholmforum.se/se/stats/presentpdf/gillespie.pdf, consulté le 3 janvier 2009

${ }^{4}$ Dans Goodness Gracious Me, le célèbre héros austenien Mr. Darcy est interprété par un personnage récurrent dans le sketch show, Chunky Lafanga, une star du cinéma bollywoodien imbue d'elle-même et désirant ardemment faire carrière en Occident. Lors de la première rencontre avec Elizabeth, ce dernier, pour la séduire, se lance dans un numéro de danse mêlant glissements dynamiques des pieds et claquements des mains (Saison 2, 
La série, en effet, ne se contentait pas de croiser les identités, elle croisait aussi les supports. Outre des séquences dignes de sitcoms, telles celles où une famille adopte une vache comme animal de compagnie, étaient incorporés des inserts vidéographiques, dont une archive d'un discours de Tony Blair en réponse à Mr. 'Have-You-MetMy-Daughter?'49, et de faux clips, le tube " Barbie Girl » étant ainsi parodié en «Punjabi Girl»50. Apte à transcrire une identité kaléidoscopique que n'aurait sans doute pas permis un format de sitcom, pareil mélange des genres semblait en même temps correspondre aux goûts d'un public désormais accoutumé à des formats plus courts et plus souples, mini-séries ou vidéodiffusion sur Internet par exemple. Très figée en termes génériques, la comédie de situation paraissait de moins en moins combler ces attentes. Quelques années plus tard, elle fut même présentée comme grotesquement désuète par l'humoriste Ricky Gervais dans la série Extras (BBC2, 2005-7) $)^{51}$.

On compte, certes, plusieurs sitcoms britanniques sudasiatiques au tournant du nouveau millénaire, All About Me (BBC1, 2002-4), dédiée à une famille recomposée et mixte, la série pour adolescents My Life as a Popat (ITV, 2004 ; 2007) ou encore Meet The Magoons $(\mathrm{C} 4,2005)$, consacrée à un restaurant punjabi de Glasgow, mais le genre est progressivement concurrencé par d'autres formats, du satirique Da Ali G Show $\left(\mathrm{C}_{4}, 2000\right)$, où Sacha Baron-Cohen mêle stand up et celebrity show, à The Kumars At $N^{\circ} 42$ (BBC2, 2001-6), une parodie de chat show inventée par Sanjeev Bhaskar. Plus de vingt ans après le lancement de The Fosters, pionnière dans l'avènement de séries humoristiques à ancrage noir en Grande-Bretagne, Goodness Gracious $M e$ semble ainsi avoir contribué à promouvoir un humour britannique-sud-asiatique à la télévision outre-Manche et à doubler l'hybridation culturelle d'une hybridation générique. Et pourtant, si ce

épisode 2, 20 novembre 1998). Six ans plus tard, dans son adaptation de Pride and Prejudice, judicieusement renommé Bride and Prejudice (2004), Gurinder Chadha reprendra cette esthétique bollywoodienne, illustrant à son tour, de façon décalée, la remarque suivante de Mr. Lucas à Darcy dans le roman original : "[Dancing] is one of the first refinements of polished societies." Jane Austen, Pride and Prejudice, chapitre 6, The Complete Novels, Londres, BCA/Crown Publishers, 1995, p. 189.

49 Saison 1, épisode 6 (16 février 1998).

${ }^{50}$ Saison 1, épisode 4 (2 février 1998).

${ }^{11}$ Dédiée aux expériences de deux figurants amenés à rencontrer diverses stars de cinéma, Extras met en abyme When the Whistle Blows, une sitcom dans laquelle joue l'un des deux héros. Ceci est l'occasion de malmener les codes du genre, en particulier à travers l'abus de rires « en boîte » et l'emploi d'une unique réplique - « Are you 'aving a laugh? » - répétée en boucle. Notons aussi que Ricky Gervais a co-écrit, avec Stephen Merchant, le populaire The Office (BBC1, 2001-3), un mock documentary, ou faux documentaire, tourné dans une fabrique de papier du Sud-Est de l'Angleterre. La série a fait l'objet d'adaptations dans divers pays, notamment en France, où Le Bureau (Canal+, 2006) n’a pas réussi à convaincre le public, et aux États-Unis où The Office US (NBC, 2005-) poursuit, en revanche, actuellement sa huitième saison. Voir l'article de Shannon Wells-Lassagne dans le présent numéro de TV/Series. 
trait persiste au vingt et unième siècle, il vient parfois servir un humour dorénavant plus exclusif et centré sur la définition d'un « Soi » " de souche».

\section{Identité nationale et comédies télévisées dans le nouveau millénaire : « une communauté de communautés »}

En 2000, sort le rapport Parekh, The Future of Multi-Ethnic Britain, qui décrit la Grande-Bretagne comme « une communauté de communautés », ajoutant qu'employés seuls

les termes «anglicité », et par extension, «britannicité » possèdent intrinsèquement une connotation raciale ${ }^{52}$.

Cette petite phrase cristallisa l'attention de plusieurs quotidiens de presse nationale; The Sun accusa le comité Parekh de "démolir la Grande-Bretagne » ("Britain Bashers »), tandis que The Telegraph et The Mail utilisèrent une accroche commune: "British' is a racist word, says report »53. La même année, suite à la publication du rapport MacPherson sur le meurtre du jeune Noir Stephen Lawrence et à sa dénonciation d'un « racisme institutionnel » policier54, fut voté le Race Relations (Amendment) Act, qui maintenait une politique de promotion de la diversité ethnique, exigeant de tout le secteur public de mettre en place des programmes annuels d' « égalité raciale 55 ». Un an plus tard, à l'été 2001, quelques mois seulement avant le choc du 11 septembre, le pays fut toutefois confronté à de nouvelles émeutes, qui opposèrent jeunes Britanniques musulmans, groupes d'extrême droite et forces de l'ordre dans le Nord de l'Angleterre. Consécutif à ces événements, le rapport Cantle marqua un changement radical en prônant la « cohésion communautaire $5^{6}$ » et en engageant les groupes minoritaires à entretenir un sentiment accru d'appartenance nationale, notamment par le biais de tests de citoyenneté, projet concrétisé en

\footnotetext{
52 « a community of communities» et «Englishness, and by extension, Britishness is racially coded. » Bhikhu Parekh, The Future of Multi-Ethnic Britain, The Parekh Report, Londres, Runnymede Trust/Profile Books, 2000, p. 37-8.

53 Pour une analyse de la polémique, voir Julian Petley, "A Case of Mistaken Identity », Index on Censorship, vol. 30, $\mathrm{n}^{\circ} 3,2001$, http://bura.brunel.ac.uk/bitstream/2438/ 4108/1/Fulltext.pdf, consulté le 27 septembre 2011, et Andrew Pilkington, " From Institutional Racism to Community Cohesion: the Changing Nature of Racial Discourse in Britain ", Sociological Research Online, vol. $13, \mathrm{n}^{\circ} 3,6$ mai 2008 , http://www.socresonline.org.uk/13/3/6.html, consulté le 26 juillet 2011.

54 William MacPherson, The Stephen Lawrence Inquiry: Report of an Inquiry by Sir William MacPherson of Cluny, Cmnd 4262-I, Londres, HMSO, 1999. Ainsi qu'on l'a vu, le " racisme institutionnel » policier fait déjà l'objet du rapport Scarman de 1981. À ce sujet, voir par exemple Stuart Hall, « From Scarman to Stephen Lawrence », History Workshop Journal, n 48 , 1999, p. 187-197.

${ }_{55}$ Race Relations (Amendment) Act, Londres, HMSO, 2000.

${ }^{5}$ Voir Ted Cantle, Community Cohesion: A New Framework for Race and Diversity, Londres, Palgrave Macmillan, 2008 [2001].
} 
novembre 2005 et en quelque sorte légitimé par les attentats londoniens de juillet. Le multiculturalisme semblait ainsi vivre ses derniers instants et même Trevor Phillips, le Président de la Commission for Racial Equality, déclarait à présent qu'ayant de facto opposé les groupes, le multiculturalisme devait tout bonnement « être mis au rebut 57 ».

Diffusée sur BBC2 de 2003 à 2006 et inventée et jouée par Matt Lucas et Dave Walliams, Little Britain semble en partie s'être nourrie de ce débat sur l'identité nationale, sur sa définition et ses limites. Ainsi que l'indiquait son titre, une combinaison de Great Britain et de Little England - qui désigne d'ordinaire les nationalistes anglais - la série sondait la psychè britannique par le petit bout de la lorgnette, se présentant comme un guide des mœurs à destination des novices ${ }^{5}$, d'où la présence d'un narrateur (Tom Baker) dès le générique, où la leçon d'anthropologie alternait entre nonsense et "fausse pub », par exemple :

Britain, Britain, Britain, everybody is welcome in Britain. We are open 9 till 6 Monday to Saturday. No foreign gentlemen please. But what makes Britain such a wonderful place to visit for an afternoon? Why! It's the people of Britain and it is these which we'll look at today. Oh my sweet Lord!59

Le sketch show continuait d'utiliser la galerie de portraits pour couvrir une variété de classes, de générations et de professions, mais aussi d'identités régionales, dont le Gallois Daffyd Thomas, alias the only gay in the village, et l'Écossais Ray McCooney, le propriétaire du Ye Olde Hotele, qui parlait occasionnellement en énigmes et jetait des sorts à ses clients ${ }^{60}$. Le nom du personnage n'était pas anodin; il s'agissait d'un hommage décalé à l'auteur Ray Cooney, parfois surnommé le «Feydeau anglais ». Cet hôtelier fou en évoquait en même temps un autre, Basil Fawlty, interprété par John Cleese dans Fawlty Towers (BBC2, 1975-79). En renouant avec un folklore comique britannique, l'intertextualité permettait sans doute à Little Britain d'inscrire le sens de l'humour lui-même dans une perspective nationale.

Or, à la différence de Fawlty Towers, où la comédie ethnique portait sur l'Espagnol Manuel (Andrew Sachs), dans Little Britain, Ray McCooney était lui-même la cible de stéréotypes relatifs à son origine.

57 Tom Baldwin, «Britain must 'scrap multiculturalism' », The Times, 3 avril 2004, http://www.timesonline.co.uk/tol/news/uk/article1055221.ece, consulté le 13 juillet 2011. $5^{8}$ Voir par exemple Renée Dickason, " Great Little Britain: Exploring the 'Other' Within », Journal of British Cinema and Television, vol. 7, août 2010, p. 248-263.

${ }^{59}$ Saison 1, épisode 4 (BBC2, 25 décembre 2003).

${ }^{60}$ Dans l'ère de l'après-dévolution, cette esthétique a été reprise par d'autres sketch shows, dont Burnistoun (BBC2 Scotland, 2010-), qui, en passant en revue les habitants d'une ville écossaise éponyme, semble à son tour révéler que l'exploration ethnographique commence aussi désormais « chez soi ». 
Dans sa tentative de définir l'identité nationale britannique, sinon anglaise, la conclusion à laquelle semblait aboutir la série était que ce qui unissait tous ces citoyens était leur rejet de l'« étranger », autrement dit, «no foreign gentlemen please». Ainsi Marjorie, animatrice pour Fat Fighters - une transposition fictionnelle du spécialiste de l'amaigrissement Weight Watchers - grossière avec l'ensemble de ses clients, s'avérait particulièrement méprisante envers Meera, une Britannique sud-asiatique, qu'elle appelait invariablement Mary et faisait mine de ne pas comprendre, tandis que Maggie, dame patronnesse du Women's Institute, participait à des concours de nourriture mais souffrait de vomissements dès qu'elle apprenait que le plat provenait d'un foyer homosexuel, noir ou sud-asiatique ${ }^{61}$. Malgré la palette d'individus dépeints, l'image de la Grande-Bretagne dans Little Britain apparaissait dès lors beaucoup plus réduite, autrement dit plus exclusivement blanche, et, précisément, plus " petite » que celle affichée dans Goodness Gracious Me trois ans auparavant.

Centré sur des Blancs, le sketch show incorporait, certes, quelques individus d'appartenance minoritaire, dont Ting Tong Macadangdang, une épouse thaillandaise (en fait un travesti) commandée via Internet par l'Anglais Dudley, mais le personnage ne servait qu'à asseoir des clichés ethniques. Fourbe et obséquieuse, Ting Tong transformait successivement le domicile conjugal en planque pour sa mère sans papier, puis en restaurant clandestin pour son frère $^{62}$. Joué par le comédien blanc Matt Lucas, le personnage de Ting Tong impliquait en outre le recours au blacking up, également utilisé par Dave Walliams pour incarner Desiree DeVere, une ex-miss Botswana obèse. De façon plus significative encore, les auteurs en usaient enfin pour interpréter deux minstrels 63 (voir figure 6), recyclant ainsi un héritage télévisé inédit depuis la fin historique des Black and White Minstrel Shows (1957-1978, voir figure 5), auxquels la BBC, sous la pression d'associations de défense des minorités, fut contrainte de mettre un terme ${ }^{64}$.

${ }^{61}$ À la suite d'une plainte, en décembre 2004, de la Federation of Women's Institutes concernant l'utilisation de leur logo, la $\mathrm{BBC}$ fut contrainte de lui trouver un substitut fictif $B B C$ News, «WI forces cuts to Little Britain », 30 décembre 2004, http://news.bbc.co.uk/2/hi/entertainment/4134671.stm, consulté le 16 mai 2011. ${ }^{62}$ Saison 3 , épisode 4 et épisode 6 (BBC2, 8 et 24 décembre 2005).

63 Mêlant danse, mime et chant, la pratique du minstrelsy consistait, pour des comédiens blancs, traditionnellement en hauts-de-forme et queues-de-pie, à se grimer le visage au cirage noir. Outre le comique visuel généré par un tel travestissement, cette convention, née aux États-Unis et exportée en Grande-Bretagne, était l'occasion de parodier l'accent de locuteurs étrangers, favorisant par là même un comique verbal. À ce sujet, voir Robert $\mathrm{C}$. Toll, Blacking Up: The Minstrel Show in Nineteenth-Century America, Londres, Oxford University Press, 1974 et Annemarie Bean et al. (éd.), Inside the Minstrel Mask: Readings in 19th Century Blackface Minstrelsy, Wesley, University Press of New England, 1996.

64 Dès mai 1967, la BBC avait reçu une pétition du groupe Campaign Against Racia Discrimination demandant que ce programme soit retiré de l'antenne. L'émission dura pendant encore plus de dix ans. Notons également la décision controversée de Bill Cotton, 


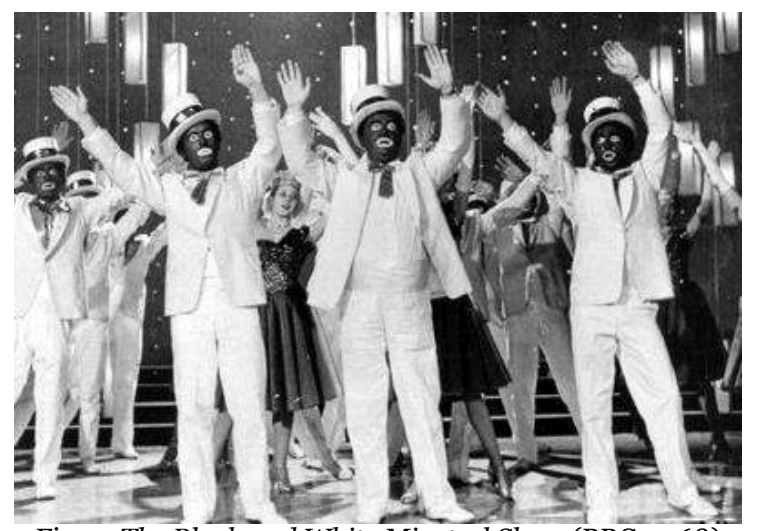

Fig. 5: The Black and White Minstrel Show (BBC, 1968)

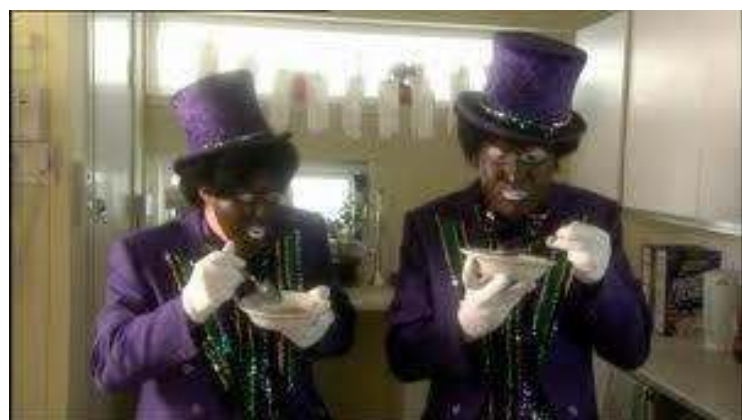

Fig. 6: Little Britain, sketch « The Minstrels », Saison 1, épisode 1 (16 septembre 2003)

Si Little Britain aborde ici brièvement la question de la discrimination - les deux minstrels sont peinés d'apprendre à la radio que, devant leur recrudescence, le Ministre de l'Intérieur désire renvoyer cette frange de la population à «Minstrel-land» -, la thématique est vite évacuée au profit d'un nouveau renvoi au programme de la BBC: la diffusion de «Swanee » sur une station de radio concurrente, soit un standard du music hall et des Black and White Minstrel Shows, sur lequel les comédiens Matt Lucas et Dave Walliams se mettent à se déhancher en cadence. Prompt à susciter la nostalgie de la plupart des téléspectateurs, susceptibles d'avoir connu,

alors Directeur général de la BBC, de ne pas diffuser d'extraits des Minstrel Shows lors du $50^{\text {ème }}$ anniversaire de la chaîne, en 1986, décevant ainsi des millions de téléspectateurs. Pour plus de détails, voir par exemple Karen Ross, Black and White Media: Black Images in Popular Film and Television, Cambridge, Polity, 2004 [1996], p. 96. 
même de seconde main, cette émission de variétés diffusée pendant plus de vingt ans, l’impression de déjà-vu qui se dégageait du sketch de Little Britain semblait correspondre à un questionnement contemporain, la série oscillant entre tradition et modernité pour entrer en résonnance avec un débat connexe à celui sur l'identité nationale - l'omnipotence du « politiquement correct ${ }^{65}$ », de plus en plus contestée sur la scène politique britannique, entre autres par le Ministre de l'Intérieur David Blunkett, qui, dans l'après-11 septembre, avait déclaré que "les immigrants devraient essayer de se sentir britanniques », ajoutant que ce dont le pays avait besoin c'était « de bon sens et non de politiquement correct ${ }^{66} »$.

En systématisant un « humour de l'humiliation ${ }^{67}$ » vis-à-vis des minorités quelles qu'elles soient, Little Britain semblait se faire le relais de cette critique, rôle alors assumé par d'autres humoristes britanniques, tel Ricky Gervais dès The Office (BBC2, 2001-2) ${ }^{68}$, puis dans Extras (BBC2, 2005-7). Ce courant de comédie a depuis fait des émules, dont Harry Enfield, qui expliqua qu'il avait décidé de reprendre sa carrière en visionnant Little Britain ${ }^{69}$. Le sketch show Harry and Paul (BBC1, 2007-), où il collabore avec Paul Whitehouse, présente lui aussi une variété de héros, dont un Nelson Mandela, qu'il incarne grimé en Noir, tour à tour dealer et pousseur de fauteuils roulants, ce qui relève, semble-t-il, d'un nouveau clin d'œil à Alf Garnett : dans In Sickness and In Health (BBC1, 1985-92), dérivée de

65 Né aux États-Unis et exporté en Grande-Bretagne, le terme peut être défini ainsi : « First used to describe an influential movement on US campuses beginning in the late 1980 s [and] appealing to the principle of affirmative action and multiculturalism [...] it promoted anti-sexist and anti-racist speech and behavior codes, which opponents denounced as illiberal. » Ian McLean and Alistair McMillan, The Concise Oxford Dictionary of Politics, Oxford, Oxford University Press, 2003, p. 414

66 BBC News, "Immigrants 'should try to feel British' ", 9 décembre 2001, http://news.bbc.co.uk/2/hi/uk news/politics/1699847.stm, consulté le 28 septembre 2011. Notons, par ailleurs, qu'en 2006 (année de diffusion de la dernière saison de Little Britain), Civitas, un groupe de réflexion de centre-droit, a publié un pamphlet contre le politiquement correct : Anthony Browne, Retreat of Reason: Political Correctness and the Corruption of Public Debate in Modern Britain, Londres, Civitas, Institute for the Study of Civil Society, 2006.

${ }^{67}$ Voir Sharon Lockyer (éd.), Reading Little Britain: Comedy Matters on Contemporary Television, Londres, I.B. Tauris, 2010, p. 5. Cet ouvrage comporte, en outre, plusieurs références d'articles de presse contemporains, issus de tabloïds et de journaux dits " de qualité », globalement très critiques à l'égard de la série, jugée offensante pour les minorités dans leur ensemble.

${ }^{68}$ L'épisode ayant attiré le plus de plaintes auprès de la BBC sur l'ensemble de la diffusion de la série reposait en partie sur une blague du patron concernant la taille du sexe des Noirs. Lorsqu'un employé noir s'approche du groupe auquel David Brent raconte la blague, ce dernier, dans une tentative de diversion, se tourne alors vers une employée en fauteuil roulant, pour demander à la cantonade " Have you met this little lady? », déplaçant ainsi le «non politiquement correct » d'une minorité à l'autre. Saison 2, épisode 1 (30 septembre 2002).

69 «Harry: My Little Brit of Fortune», The Sun, 27 septembre 2010. http://www.thesun.co.uk/sol/homepage/showbiz/tv/3154027/Harry-My-Little-Brit-offortune.html, consulté le 12 juin 2011. 
Till Death Us Do Part, ce héros raciste, désormais impotent, était contraint d'engager un aide à domicile, noir et homosexuel, qui le promenait en fauteuil.

Depuis le nouveau millénaire, plusieurs séries humoristiques britanniques ont ainsi opéré une sorte de retour en arrière et à l'heure où la Grande-Bretagne s'interroge encore sur la définition et sur les limites d'une identité nationale ${ }^{70}$, il ne paraît pas anodin qu'elles puisent leur inspiration dans des situations ethniques. Au moment de la rédaction de cet article, la tendance ne semble pas s'inverser; en janvier 2011, la BBC a commandé à Matt Lucas et Dave Walliams une seconde saison de Come Fly With Me (BBC1, 2010-), un mock documentary tourné dans un aéroport et qui recycle non seulement le blacking up, mais une kyrielle de stéréotypes raciaux ${ }^{71}$. Plus de dix ans après la publication du rapport Parekh, la description controversée de la société britannique comme "une communauté de communautés » semble d'ailleurs également symptomatique en regard du paysage audiovisuel en matière de comédie. Parallèlement à Come Fly With Me, la BBC programme des séries telles The Omid Djalili Show (20072009), où le comédien britannique d'origine iranienne ironise, entre autres, sur la guerre du gouvernement contre le terrorisme, ou, prochainement, The Imran Yusuf Show, qui mettra en scène un Britannique musulman né au Kenya et ayant grandi à Londres. En 2000, le Directeur général de la BBC, Greg Dyke, avait qualifié la chaîne d' "affreusement blanche ${ }^{72}$ », constat réitéré en 2010 par le Directeur du BBC College of Comedy, Micheal Jacob, qui la jugeait " trop blanche et trop classe moyenne ${ }^{73}$ ». Ceci tendrait à indiquer qu'en matière de relations raciales, comme à la télévision, le débat britannique entre « majorité » et « minorités » a finalement peu évolué au cours de la dernière décennie.

\footnotetext{
${ }^{70}$ En février 2011, le Premier Ministre David Cameron soulignait encore la nécessité que la Grande-Bretagne développe une identité nationale forte pour prévenir toute forme de terrorisme parmi ses citoyens. Voir par exemple Oliver Wright et Jerome Taylor, "Cameron: My war on multiculturalism ", The Independent, 5 février 2011, http://www.independent.co.uk/news/uk/politics/cameron-my-war-on-multiculturalism2205074.html, consulté le 28 septembre 2011.

${ }_{71} \mathrm{La}$ série a fait couler beaucoup d'encre et la décision de la $\mathrm{BBC}$ a donc été controversée. Voir par exemple Balaji Ravichandran, "Come Fly With Me is tasteless comedy », The Guardian, 28 décembre 2010, www.guardian.co.uk/commentisfree/2010/dec/28/comefly-with-me, consulté le 28 septembre 2011, et Simon Cable, «Viewers protest at Matt Lucas and David Walliams's 'minstrel show': Comedy duo's new BBC spoof accused of being racist ", Mail online, 28 décembre 2010,

www.dailymail.co.uk/tvshowbiz/article1342025/David-Walliams-Matt-Lucas-BBC-spoofCome-Fly-With-Me-accused-racism.html, consulté le 28 septembre 2011.

${ }_{72}$ Voir note 23 du présent article.

73 Voir note 1 du présent article.
} 


\section{Bibliographie}

Amin Kaushika et Carey OpPenheIm, Poverty in Black and White: Deprivation and Ethnic Minorities, Londres, Child Poverty Action Group/Runnymede Trust, 1992.

ANWAR Michael et Anthony SHANG, Television in a Multi-Racial Society, A Research Report, Londres, Commission for Racial Equality, 1982.

BALDWIN Tom, «Britain must 'scrap multiculturalism' », The Times, 3 avril 2004, www.timesonline.co.uk/tol/news/uk/article1055221.ece, consulté le 13 juillet 2011.

BARTHES Roland, "L'Effet de réel ", in Littérature et réalité, éd. Gérard Genette et Tzvetan Todorov, Paris, Seuil, 1982, p. 88-89 [p. 8190].

$B B C$ News, "WI forces cuts to Little Britain ", 30 décembre 2004, http://news.bbc.co.uk/2/hi/entertainment/4134671.stm, consulté le 16 mai 2011.

BEAN Annemarie et al. (éd.), Inside the Minstrel Mask: Readings in 19th Century Blackface Minstrelsy, Wesley, University Press of New England, 1996.

Browne Anthony, Retreat of Reason: Political Correctness and the Corruption of Public Debate in Modern Britain, Londres, Civitas, Institute for the Study of Civil Society, 2006.

CABLE Simon, "Viewers protest at Matt Lucas and David Walliams's 'minstrel show': Comedy duo's new BBC spoof accused of being racist ", Mail online, 28 décembre 2010, www.dailymail.co.uk/tvshowbiz/article1342025/David-WalliamsMatt-Lucas-BBC-spoof-Come-Fly-With-Me-accused-racism.html, consulté le 28 septembre 2011.

CANTlE Ted, Community Cohesion: A New Framework for Race and Diversity, Londres, Palgrave Macmillan, 2008 [2001].

COHEN Robin, Frontiers of Identity, The British and the Others, Londres, Longman, 1994.

CoHEN Tamara, «BBC launches competition for multicultural writers as its sitcoms are branded 'too middle class' ", Daily Mail online, 20 août 2010, www.dailymail.co.uk/tvshowbiz/article-1304608/BBC- 
competition-multicultural-writers-sitcoms-branded-middle-class.html, consulté le 7 septembre 2011

DiCKASON Renée, "Great Little Britain: Exploring the 'Other' Within' ", Journal of British Cinema and Television, vol. 7, août 2010, p. 248-263.

DuCRAY Amandine, Entretien avec Vince Powell, Londres, $1^{\text {er }}$ août 2005, in Amandine Ducray, "Les Sitcoms ethniques à la télévision britannique (1972-1998)» (Appendices, vol. 2), Thèse de doctorat dirigée par Renée Dickason et soutenue à l'Université de Caen le 5 décembre 2005, p. 589-598.

DuCRAY Amandine, «Ethnic Minorities, Comedy and British Television. Interview with Sharat Sardana. London, Friday 26 of November 2004", Revue LISA/LISA e-journal, http://lisa.revues.org/index664.html, consulté le 2 août 2011.

Eaton Mick, «Television Situation Comedy", Screen, vol. 19, $\mathrm{n}^{\circ} 4$, 1978-79, p. 61-89.

Equity's Policy Statement on Integrated Casting, Londres, Equity, 1983.

FEUER Jane, «Situation comedy: part two », in The Television Genre Book, éd. Glen Creeber, Londres, British Film Institute, 2001, p. 69-70.

Fonds d'action et de soutien pour l'intégration et la lutte contre les discriminations, Écrans pâles? Diversité culturelle et culture commune dans l'audiovisuel, Paris, La Documentation française, 2004.

FoucAult Michel, «Des espaces autres » (conférence au Cercle d'études architecturales, 14 mars 1967), Architecture, Mouvement, Continuité, ${ }^{\circ} 5$, octobre 1984, p. 46-49.

GEDDES Andrew, The Politics of Immigration and Race, Manchester, Baseline Books, 1996.

GILROY Paul, « C4 Bridgehead or Bantustan? », Screen, juillet-octobre 1983, reproduit in Black Images in British Television, The Colour Black, éd. Therese Daniels et Jane Gerson, Londres, British Film Institute, 1989, p. 40-43.

There Ain't No Black in the Union Jack, Londres, Routledge, 2002 [1987]. 
HaLL Stuart, «From Scarman to Stephen Lawrence », History Workshop Journal, nº48, 1999, p. 187-197.

Намамото Darrell Y., Nervous Laughter, Television Situation Comedy and Liberal Democratic Ideology, New York, Praeger, 1991 [1989].

Hepple Bob, « Have Twenty-five Years of Race Relations Acts in Britain been a Failure? ", in Discrimination: The Limits of the Law, éd. Bob Hepple et Erika Szyszczak, Londres, Mansell, 1992, p. 284-291.

Journal of the Royal Television Society (The), "A new kind of laughter », janvier 2000.

Knowles Elizabeth (éd.), The Oxford Dictionary of Phrase and Fable, Oxford, New York, Oxford University Press, 2001.

LEWISOHN Mark, Radio Times Guide to TV Comedy, Londres, BBC Worldwide Ltd, 2003 [1998].

LOCKYER Sharon (éd.), Reading Little Britain. Comedy Matters on Contemporary Television, Londres, I.B. Tauris, 2010.

MACPherson William, The Stephen Lawrence Inquiry: Report of an Inquiry by Sir William MacPherson of Cluny, Cmnd 4262-I, Londres, HMSO, 1999.

MALIK Sarita, Representing Black Britain: Black and Asian Images on Television, Londres, Sage, 2002.

McLean Ian et Alistair McMillan (éd.), The Concise Oxford Dictionary of Politics, Oxford, Oxford University Press, 2003.

NeALe Steve et Frank Krutnik, Popular Film and Television Comedy, Londres, Routledge, 1995 [1990].

PAREKH Bhikhu, The Future of Multi-Ethnic Britain, The Parekh Report, Londres, Runnymede Trust/ Profile Books, 2000.

Petley Julian, "A Case of Mistaken Identity », Index on Censorship, vol. $30, \mathrm{n}^{\circ} 3,2001$, http://bura.brunel.ac.uk/bitstream/2438/4108/1/ Fulltext.pdf, consulté le 27 septembre 2011.

PILKIngton Andrew, "From Institutional Racism to Community Cohesion: the Changing Nature of Racial Discourse in Britain ", Sociological Research Online, vol. 13, $\mathrm{n}^{\circ} 3,6$ mai 2008, 
http://www.socresonline.org.uk/13/3/6.html, consulté le 26 juillet 2011.

PINES Jim (éd.), Black and White in Colour: Black People in British Television Since 1936, Londres, British Film Institute, 1992.

Race Relations (Amendment) Act, Londres, HMSO, 2000.

RAVICHANDRAN Balaji, "Come Fly With Me is tasteless comedy », The Guardian, 28 décembre 2010, http://www.guardian.co.uk/ commentisfree/2010/dec/28/come-fly-with-me, consulté le 28 septembre 2011.

Ross Karen, Black and White Media: Black Images in Popular Film and Television, Cambridge, Polity, 2004 [1996].

SCARman Lord, The Brixton Disorders 10-12 April 1981, Cmnd 8427, Londres, HMSO, 1981.

SwanN Lord, Education for All: Report of the Committee of Inquiry into the Education of Children from Ethnic Minority Groups, Cmnd 9453, Londres, HMSO, 1985.

Toll Robert C., Blacking Up: The Minstrel Show in NineteenthCentury America, Londres, Oxford University Press, 1974.

Twitchin John (éd.), The Black and White Media (Show) Book, Handbook for the Study of Racism and Television, Stoke-on-Trent, Trentham Books, 1992 [1988].

WRIGHT Oliver et Jerome TAYLOR, «Cameron: My war on multiculturalism ", The Independent, 5 février 2011, http://www.independent.co.uk/news/uk/politics/cameron-my-waron-multiculturalism-2205074.html, consulté le 28 septembre 2011. 